\title{
Productividad potencial del SAF cacao asociado con árboles forestales
}

\section{SAF cocoa productivity potential associated with forest trees}

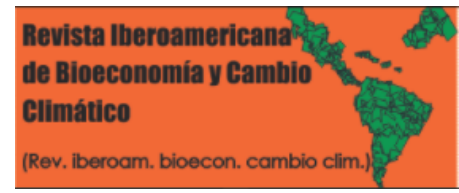

\author{
Sol-Sánchez, Ángel; López-Juárez, Sergio Alexander; Córdova- \\ Ávalos, Víctor; Gallardo-López, Felipe; Editor Académico Prof. Dr. \\ Carlos Alberto Zúniga-González
}

\author{
Ángel Sol-Sánchez \\ sol@colpos.mx \\ Colegio de Postgraduados- Campus Tabasco., México \\ Sergio Alexander López-Juárez \\ sergio.lopez@colpos.mx \\ Colegio de Postgraduados- Campus Tabasco., México \\ Víctor Córdova-Ávalos \\ Colegio de Postgraduados- Campus Tabasco., México \\ Felipe Gallardo-López \\ Colegio de Postgraduados- Campus- Veracruz., México \\ Editor Académico Prof. Dr. Carlos Alberto Zúniga- \\ González \\ Universidad Nacional Autónoma de Nicaragua, León. \\ Escuela de ciencias agrarias y veterinarias., Nicaragua
}

Revista Iberoamericana de Bioeconomía y Cambio

Climático

Universidad Nacional Autónoma de Nicaragua, León, Nicaragua

ISSN-e: 2410-7980

Periodicidad: Semestral

vol. 4, núm. 7, 2018

czuniga@ev.unanleon.edu.ni

Recepción: 01 Enero 2018

Aprobación: 18 Julio 2018

\section{URL: http://portal.amelica.org/ameli \\ jatsRepo/394/3941754011/index.htm}

DOI: https://doi.org/10.5377/ribcc.v4i7.6327

Autor de correspondencia: sergio.lopez@colpos.mx
Resumen: El objetivo de la investigación fue estimar el ingreso económico en un sistema cacaotal a partir de la sustitución de árboles de sombra muertos por árboles de pimienta y pronosticar el comportamiento del agroecosistema cacaotal al enriquecimiento con especies de alto valor comercial. Se realizó la investigación en los predios El Cometa y La Ceiba ubicados en el municipio de Comalcalco, Tabasco. En el sitio El Cometa, se registró la cantidad de árboles de pimienta, en cada una de ellas se midieron el diámetro a la altura del pecho, altura total, fuste limpio y se registró la cantidad de producción por árbol. Además, se registró la última producción de la cosecha obtenida para el cacao. En el segundo sitio, La Ceiba, se registró la cantidad de árboles forestales asociadas con el sistema agroforestal cacao. Para cada árbol se midió el DAP, altura total y fuste limpio. Con esto se obtuvo el área basal por individuo, para luego calcular el volumen comercial (vc) total de cada especie. Obtenido el vc (m3r) se obtuvo el precio de cada especie para ser colocado en el mercado. Cedrela odorata (L.) Gaertn, Swietenia macrophylla King y Ceiba pentandra (L.) Gaertn., estas fueron las especies que registran un mejor valor en el mercado de la madera nacional e internacional.

Palabras clave: Arboles, Cacaotal, Sombra, Valor comercial.

Abstract: The objective of the research was to estimate the income in a cacao plantation system from replacing dead shade trees and pepper trees predict the behavior of cacao agroecosystem enrichment with species of high commercial value. The research was conducted on the premises Comet and La Ceiba located in the city of Comalcalco, Tabasco. At El Cometa, the amount of pepper trees in each diameter at breast height, total height, the clean shaft was measured and the amount of production per tree was recorded was recorded. In addition, the latest crop production obtained for cocoa was recorded. In the second place, La Ceiba, the amount of forest trees associated with cocoa agroforestry system was recorded also. For each tree DBH, total height and the clean shaft was measured. With that he pulled the basal area per individual, and then, through a formula to get the total trade volume (cc) of each species. calculated the commercial volume $(\mathrm{m} 3 \mathrm{r})$ the price of each species to be placed on the market was investigated. Cedrela odorata (L.) Gaertn, Swietenia macrophylla King and Ceibapentandra (L.) Gaertn., These species recorded better value in the market of national and international wood. 
Keywords: Cacao trees, Shade, Market value.

\section{INTRODUCCIÓN}

La situación que atraviesan los bosques y selvas del mundo es preocupante y difícil, debido a múltiples factores, como la tala inmoderada, el incremento de la frontera agrícola, la ganadería extensiva, el cambio climático, los incendios forestales, las inundaciones y el crecimiento de la población, lo que ha repercutido en la degradación y pérdida de tierras (FAO, 2014). En México, se registró una pérdida de 1309,000 ha en el periodo comprendido de 1980 al 2000 (FAO, 2005).

A medida que el mundo se enfrenta a tasas alarmantes de pérdida de selvas tropicales, algunos sistemas agrícolas ofrecen una alternativa para frenar y amortiguar el desastre. En particular, el sistema agroforestal (SAF), el cual consiste en el manejo adecuado de tierras e implica la asociación de árboles forestales con cultivos o ganadería, o una combinación de ambos, tal asociación puede ser simultánea o escalonada en el tiempo de acuerdo al terreno. Por esa razón los SAF's surgen como una estrategia para disminuir los problemas ecológicos, sociales, ambientales y económicos en zonas rurales. Además, estos sistemas constituyen una forma eficaz de mejorar la resistencia de la agricultura y amortiguar las fluctuaciones en el microclima (Siles et al., 2010). Los agricultores, a través de los sistemas agroforestales, enfrentan el desafío de aumentar su productividad evitando la deforestación (Banco Mundial, 2012).

El SAF se destaca de otros sistemas por tres aspectos esenciales: (i) su funcionamiento se basa en las relaciones existentes entre las especies (competencia, facilitación), (ii) ofrecen una alta diversidad biológica, y (iii) producen una multiplicidad de bienes y servicios ambientales que los monocultivos no ofrecen. Estudios muestran que no solo la diversidad de árboles forestales dentro de un SAF cacao es importante, sino también la amplia variedad de aves, mamíferos no voladores e invertebrados (Ibarra et. al., 2001).

El cultivo del cacao (Theobroma cacao L.) se practica en los sistemas agroforestales. En Latinoamérica, el cultivo se ha realizado bajo la sombra de especies arbóreas provenientes del bosque natural, que en muchas ocasiones, no aportan un valor económico para los productores, pero si un valor biológico. Sin embargo, en los últimos tiempos se ha venido introduciendo nuevas especies de árboles de sombra de usos múltiples: alimenticio, maderables, combustibles, entre otros (Ospina, 2002, Sotomayor et al., 2008; Ramírez, 2009). El SAF's de cacao es muy común en el sureste de México, en donde los árboles forestales proporcionan sombra, productos comerciales y otros. El cacao, después del café, es el cultivo más importante bajo sombra en el trópico mexicano (González, 2005). En México, el cultivo de cacao se encuentra bajo el dosel arbóreo formado por árboles seleccionados de sombras como frutales y maderables (Bárcenas y Ordoñez, 2008).

Los árboles dentro del SAF prestan servicios ecosistémicos: modifican el régimen de luz (tanto en calidad como en cantidad), la temperatura del aire, humedad y movimiento del aire dentro de la plantación, que influye directamente en la fotosíntesis, crecimiento y producción de cacao (De Almeida y Valle, 2007; Zuidema et al., 2005). Asimismo, favorecen o dificultan la dinámica poblacional y la incidencia de plagas y enfermedades (y de sus enemigos naturales) que reducen los rendimientos tanto de cacao y de las especies involucradas (Pérez-Salicrop et al., 2008), producen cantidades significativas de materia orgánica, reciclaje de nutrientes y ayudan a mantener la fertilidad natural del lugar.

Lo que es importante en cultivos, como en el cacao, que se utiliza la práctica agronómica de la fertilización, y la producción de bienes y servicios (madera, leña, frutos, resinas, medicina, etc.) y servicios (los valores estéticos y culturales, la conservación de la biodiversidad, el suelo y el agua, almacenamiento de carbono

\section{Notas DE AUTOR}


atmosférico y mitigación del cambio climático, etc.) para los hogares y la sociedad global (Schroth y Harvey 2007).

La selección de especies de sombra dentro de los cacaotales, habitualmente obedece a la búsqueda de beneficios socioeconómicos y de manejo para la obtención de productos rentables (madera, leña, fruta, etc.), baja competencia con el cultivo principal, disminución de plagas o enfermedades y disminución de la erosión del suelo (Vega 2005).

Los productores de cacao reconocen que la baja productividad es el principal problema al que se enfrentan y esto lo atribuyen a diferentes causas que se clasifican en socioeconómicas, técnicas y biológicas (Dormon et al., 2004).

Para incentivar la productividad en los cacaotales se buscó sustituir árboles muertos (no productivos) por árboles productivos o útiles como la pimienta (Pimienta dioica L. Merril), la cual tiene muchas propiedades y usos en la industria alimentaria y perfumería (CONAFOR, 2014; Córdova et al., 2001).

\section{Pimienta Gorda}

La pimienta gorda (Pimienta dioica L. Merrill) es originaria de México y Centroamérica, ha sido domesticada y naturalizada en varios países tropicales del mundo. En México, ecológicamente se distribuye en la vertiente del Golfo de México, desde el norte de Puebla y Veracruz hasta el sur de la Península de Yucatán (García, 1971). Esta especie puede cultivarse en sistemas agroforestales, se le encuentra asociado principalmente en plantaciones de cacao y coco (Córdova et al., 2001).

La pimienta juega un papel importante en el rubro económico, tiene presencia en todos los continentes y el valor de venta asciende a 1000 millones de dólares, especialmente en países de la Unión Europea y Estados Unidos (Claridades Agropecuarias, 2001). Para el año 2011, se enviaron al mercado internacional 312,717 toneladas; Vietnam fue el principal exportador con el 39.6\% del total. Así mismo, Indonesia, Brasil, India y Malasia, con el 11.7\%, 10.5\%, 12\% y 4.6\% respectivamente. En este rubro, México ocupa el lugar 13, con el 1.9\%, al haber exportado 5,998 toneladas (FAOSTAT, 2014). La FAO (2014) indica que en el año 2011, se compraron 223,966 toneladas a nivel mundial, de los cuales Estados Unidos importó 30.6\% a nivel mundial, Alemania, Países Bajos, India y Singapur, con una participación de 11.4\%, 8.2\%, 6\% y 5.3\% respectivamente.

La producción total de este cultivo, para el año 2012, fue de 455,768 t. Los principales países productores fueron Vietnam, Indonesia, India, Brasil, China, Malasia y Sri Lanka. Vietnam, Indonesia e India, los cuales destacaron por su nivel de producción, estos países obtuvieron el 64.6\% del total. Por su parte, México ocupó el lugar 13 al aportar apenas el $0.66 \%$ de la producción mundial, La superficie establecida para el año 2013 fue de 3,381.50 hectáreas y los principales estados productores fueron Guerrero, Tabasco y Chiapas (SIAP, 2014).

\section{SISTEMAS AGROFORESTALES}

En países de América Latina, se ha encontrado que la especie Gliricidia sepium Jacq., se ha utilizado como árbol de sombra para el cacao ya que incrementa la entrada de nitrógeno al sistema, Tscharntke et al., (2011), cita que esta especie en cacaotales se captura en promedio $30 \mathrm{Kg} / \mathrm{ha}-1 /$ año de $\mathrm{N}$. En Talamanca, Costa Rica se recomienda una determinada especie forestal dependiendo de la fertilidad del suelo, para suelos con buena fertilidad y bien drenados, se recomienda laurel (Cordia alliodora), y para aquellos con drenaje deficiente el macuili (Tabebuia rosea Bertol) (Somarriba y Beer, 1999). Asimismo, en la reserva indígena Bribri de Talamanca, Costa Rica se reportó el uso de 29 especies de árboles de sombra asociadas con cacao. En Tabasco y Chiapas se ha descrito la diversidad de árboles asociados al cacaotal, algunos útiles en la alimentación y otros productores de madera (Córdova et al., 2001; Ramírez, 2009, Roa-Romero et al., 2009), y particularmente 
para Tabasco se reportan que las principales especies son Erythrina americana Mill, Diphysa robinoides Benth, Gliricidia sepium (Jacq.) Walp, Samanea saman (Jacq.) Merr. y Colubrina arborescens (Mill.) Sarg., por su abundancia (Ramos, 2001).

Por otro lado, Sánchez et al., (2012) registro en plantaciones de cacao de Tabasco 67 especies maderables, siendo las más representativas el cedro (Cedrela odorata), cocoite (Gliricidia sepium), eritrina (Erythrina americana), macuili (Tabebuia rosea) y chipilcoite (Diphysa robinioides), mientras que Roa-Romero et al., (2009) en el Soconusco Chiapas, señalan 46 especies arbóreas pertenecientes a vegetación nativa y secundaria como el zapote (Pouteria sapota Jacq.) Moore \& Stearn, mango (Mangifera indica L.), laurel (Cordia alliodora Ruiz y Pav. Oken, cedro (Cedrela odorata L.) y cocotero (Cocos nucifera L.).

En ese sentido el objetivo de esta investigación fue estimar el ingreso económico en un sistema agroforestal cacao remplazando los árboles de sombra muertos por árboles de pimienta y especies de alto valor comercial.

\section{Materiales y MÉTODOS}

\section{Área de estudio}

La investigación se realizó en el ejido Francisco I. Madero, Segunda Sección del municipio de Comalcalco, Tabasco; México en la región de la Chontalpa, ubicado en las coordenadas $18^{\circ} 15^{\prime} 42.15^{\prime \prime} \mathrm{LN}$ y 93⒉'5.92" LW a una altitud de 0-10 $\mathrm{msnm}$. Presenta clima cálido húmedo con abundantes lluvias en verano, con precipitación anual de $1500-2500 \mathrm{~mm}$; y con temperatura de $28 \pm 30^{\circ} \mathrm{C}$. Colinda al norte con el municipio de Paraíso, al este con los municipios de Paraíso y Jalpa de Méndez; al sur con los municipios de Jalpa de Méndez, Cunduacán y Cárdenas, al oeste con el municipio de Cárdenas. La extensión territorial del municipio es de $723.19 \mathrm{~km} 2$, sobre el cual se asientan 90 rancherías y 30 ejidos (INEGI 2012) (Figura 1). Los sitios de estudio fueron los predios El Cometa y La Ceiba.

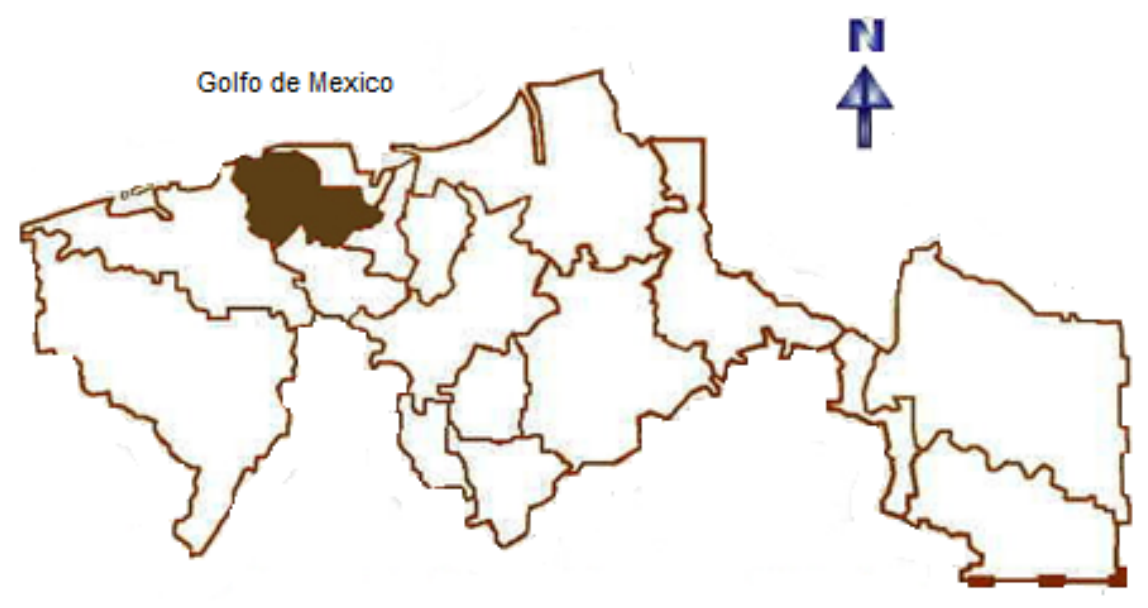

FIGURA 1.

Localización de los sitios de El Cometa y La Ceiba en el municipio de Comalcalco, Tabasco. INEGI 2012 


\section{MEDiCión DE VARIABLES}

Se seleccionaron dos plantaciones de cacao, con una edad, superficie y producción homogéneas. En ambas parcelas se registró el nombre del productor, nombre del predio, superficie de la parcela, edad de la plantación del cacao y se georreferenciaron con un GPS (Garmin modelo GSmap60csx) y se registraron las fechas de la última cosecha de cacao.

En el primer sitio, El Cometa (de una hectárea), se contabilizó el número total de árboles de pimienta, se registró el diámetro a la altura del pecho (DAP) con una cinta diamétrica y la altura con una pistola haga ${ }^{\circ}$ y se registró la producción de pimienta por árbol.

En el segundo sitio, La Ceiba (dos hectáreas), se registraron las especies forestales presentes y se calcularon el ingreso económico a partir de la venta de madera. A cada árbol forestal se le midió el diámetro a la altura del pecho (DAP) con una cinta diamétrica, fuste limpio y altura total se midieron con una pistola haga ${ }^{\bullet} \mathrm{y}$ se calculó el volumen comercial de la madera

\section{ANÁLISIS ESTADÍSTICo}

La información generada se analizó mediante un análisis de varianza para un diseño completamente al azar, usando el PROC GLM de SAS versión 9.4 y para las comparaciones de medias se empleó la prueba Tukey con $\mathrm{p}<0.05$.

\section{ReSUltados Y Discusión}

En el sitio "El Cometa" se registraron 40 árboles de pimienta, El árbol con menor DAP fue de $17 \mathrm{~cm}$ y el mayor con $145 \mathrm{~cm}$; la menor altura fue de $7 \mathrm{~m}$ y el mayor con $22 \mathrm{~m}$. En relación a la producción, tres árboles produjeron más de $10 \mathrm{~kg}$ de pimienta, pero menos de $13 \mathrm{~kg}$ cada uno; a seis árboles se les cosecho entre $8 \mathrm{y}$ $9.9 \mathrm{~kg}$, mientras que en nueve arboles su producción fue

alrededor de $7 \mathrm{~kg}$. De los restantes, 10 produjeron seis $\mathrm{kg}$ en promedio cada uno y 12 produjeron cinco $\mathrm{kg}$ o menos. En relación al diámetro tres arboles registraron más de $100 \mathrm{~cm}$ de diámetro, ocho arboles entre $50 \mathrm{y}$ 99 y los 29 restantes tuvieron un diámetro menos de $50 \mathrm{~cm}$. Esta relación de diámetros indica que los arboles de pimienta no son tan viejos como la plantación de cacao, y que su producción no se encuentra al máximo.

\section{Producción cacao}

Para la última cosecha, se obtuvo una producción de $80 \mathrm{~kg}$ de cacao en seco por hectárea y en este periodo, como la pimienta apenas había iniciado floración y fructificación su producción promedio de pimienta fue de $6.5 \mathrm{~kg}$ por árbol. El productor realiza el beneficio de la pimienta de acuerdo a sus necesidades. Para que la asociación de pimienta y cacao sea rentable. Sánchez y Dubón (1993) cita que se requiere al menos 91 árboles de pimienta/ha, mientras que Marcos (2006) hace mención que se necesitan, como mínimo 71 plantas de pimienta/ha. Sin embargo, el INIFAP (2011) indica que 40 árboles son suficientes para hacer rentable tal asociación. Esta recomendación es similar a lo registrad en este estudio. Este aspecto debe analizarse puesto que la pimienta es de follaje espeso que no permite la entrada de luz que podría afectar la producción de caco, por lo que habría que evaluar cuantos arboles por hectárea de caco es lo más aceptable.

Durante el estudio el precio del cacao en baba y en seco se encontraba en 23 y 36 pesos. Mientras que la pimienta verde y seca se encontraba en 14 y 32 pesos, respectivamente. La mayor producción de la pimienta inicia a partir del noveno año con un rendimiento por árbol de $18 \mathrm{~kg}$ (INIFAP, 2011) pero esto depende de la cobertura de la copa, y el precio por $\mathrm{kg}$ es 32 pesos, por tanto, se podrá obtener un ingreso extra por 
la venta de la pimienta. Puesto que los árboles de pimienta no se encuentran en su máxima producción el ingreso igualmente no es el mayor, este tiende a aumenta al hacerse más viejos los árboles y extender sus copas. Sol (1999), cita que, en áreas abiertas como potreros, la copa de los árboles de pimienta se extiende desde temprana edad y su producción se acelera generando volúmenes mayores que áreas sombreas aunque los frutos son de menor tamaño.

A pesar de que la pimienta es considerada como un cultivo alternativo, su precio llega a ser más alto que el de los cultivos principales con los que se asocia y esto representa una importante fuente de ingreso para el productor. La asociación en el cultivo genera ingresos económicos y promueve una mayor diversidad biológica, mejora el uso de los recursos naturales, reduce la erosión de suelos, disminuye el riesgo de pérdida total de cosecha y existe menor riesgo por plagas y enfermedades (Francis, 1990).

\section{ÁrbOLES FORESTALES}

Para el segundo sitio se analizó una superficie de 2 hectáreas de cacao y se obtuvo una producción de 120 $\mathrm{kg}$ en seco. En el inventario forestal se registraron 324 árboles, pertenecientes a 25 especies agrupados en 17 familias. Las especies que registraron mayor número de individuos fueron cedro (Cedrela odorata L.), macuili (Tabebuia rosea Bertol DC.), cocoite (Gliricidia sepium Jacq. Steud.), caoba (Swietenia macrophylla King.) y guácimo (Guazuma ulmifolia L.); éstas representaron el 51.5\% del total de los árboles registrados.

Las familias más diversas fueron: Meliaceae (3 especies y 79 individuos), Fabaceae (4 especies y 71 individuos en), Bignoniaceae (43 individuos), Anacardiácea 22 y Malvaceae (21 individuos). Las demás especies estuvieron representadas por un menor número de individuos.

Las especies que registraron mayores individuos son el cedro (Cedrela odorata L.) con 44 individuos (13.6\% del total de especies), seguido por macuili (Tabebuia rosea Bertol DC) con 43 individuos (13.3\% del total de especies), cocoite (Gliricidia sepium Jacq) con 36 individuos (11.1\% del total de especies), caoba (Swietenia macrophylla) con 24 individuos (7.4\% del total de especies), guácimo (Guazuma ulmifolia) con 20 individuos (6.2\% del total de especies) y eritrina (Erythrina poeppigiana) con 17 individuos (5.2\% del total de especies) (Figura 2).

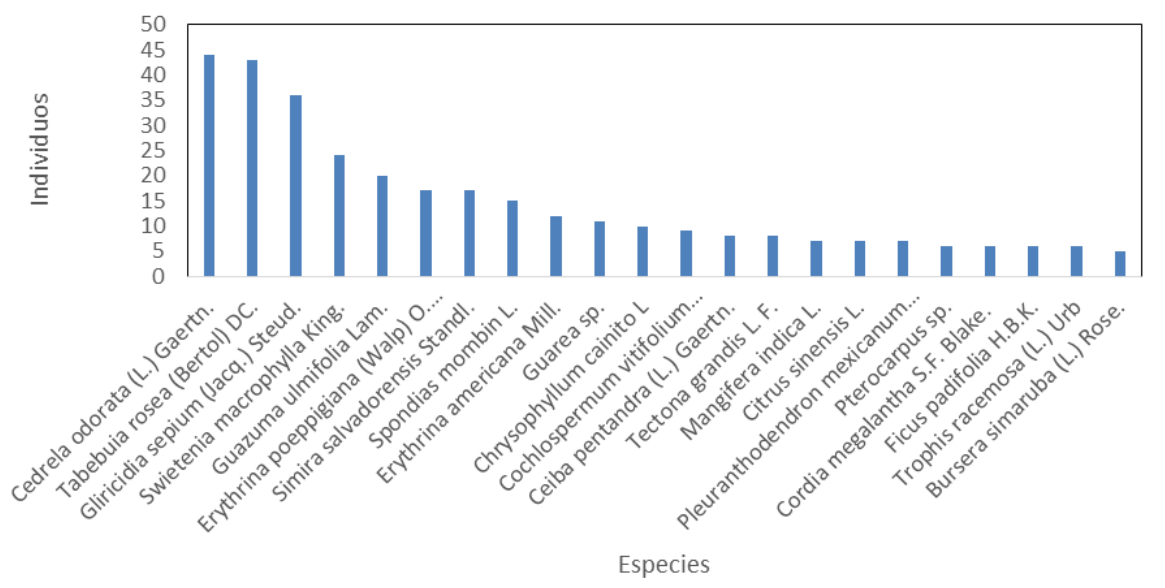

FIGURA 2

Especies registradas en el sistema agroforestal cacao, el sitio La Ceiba, Comalcalco; Tabasco.

En el inventario forestal se obtuvieron especies con alto valor comercial en el mercado. (Beer et al., 1998) menciona que el cedro (Cedrela odorata) es una de las especies maderables más importantes para la sombra del café en América Latina, mientras que en Panamá estudios demuestran que el cedro junto con la pochota (Pachira quinata) y macuili (Tabebuia rosea) son consideradas especies maderables de alto valor empleadas 
por productores de café y cacao (Jefferson et al., 2011). En la parcela de estudio, se registraron 43 individuos de macuilí la cual sirve como sombra para el cacao y reduce la erosión. El cocoite (Gliricidia sepium Jacq. Steud.) es una especie usada para leña, postes y artesanías; tiene muchos propósitos y genera ingresos extras al productor (Bear et al., 2004; Vázquez-Yánes et al., 1999). También, se ha propuesto esta especie para sustituir especies sin valor comercial ya que se comercializan localmente para postes aserrados y también puede ser utilizado para restaurar y aumentar fertilidad de suelos.

La caoba (Swietenia macrophylla King) es una especie demandante puesto que su madera es la más valiosa del mundo y mediante plantaciones ha sido útil para contrarrestar la extracción de áreas naturales. La madera de la caoba es una fuente de ingresos para pequeños productores y la creciente demanda ha propiciado que esta especie haya sido reducida severamente en su área de distribución, por lo que se ha asociado al cultivo de cacao desde la antigüedad.

El cacao asociado con cedro (Cedrela odorata L. Gaertn), macuili (Tabebuia rosea Bertol DC.), cocoite (Gliricidia sepium Jacq. Steud.) y otras especies forestales, favorece a que el productor mayores posibilidades de satisfacer sus necesidades de madera, leña o ingreso por venta. Por otra parte, se obtienen frutas de consumo familiar y comercio local como el chinin (Persea shiedeana) y mango (Mangifera indica).

Los ingresos se ven mejorados con la incorporación de una gran diversidad de especies de árboles útiles las plantaciones de cacao, a su ve esta asociación con árboles en cacaotales y café reduce costos de producción (Murgueitio et al., 2011). La producción de madera es una función importante para los pequeños productores en los Sistemas agroforestales de cacao ya que dependiendo de las especies maderables, tiende aumentar el ingreso económico haciendo más productivo el sistema. La asociación de árboles frutales y forestales con cacao trae condiciones propicias para conservar la biodiversidad (Griffith, 2000) y sirven como refugios para la fauna (Greenberg et al., 2000). Además, funcionan como corredores biológicos para el movimiento de animales y dispersión de vegetales entre los fragmentos de sistemas.

Los sistemas agroforestales de cacao en América Central albergan una alta riqueza de especies de flora maderable y alta complejidad estructural (Deheuvels et al., 2012), aumenta la diversidad de productos para el autoconsumo y la venta crece (Dalquist et al., 2007). Para reducir la deforestación y la pérdida de la producción, se han realizado estudios proponiendo transformar la sombra improductiva en sombra productiva del cacao, esto genera una intensificación de nuevas especies arbóreas y aumenta la complejidad de biodiversidad (Gockowski y Sonwa, 2011). En Bahía, Brasil se implementó la asociación de árboles forestales con cacao, esto permitió incrementar la biodiversidad, incremento la captura de carbono y acrecentó la productividad del cultivo del cacao.

Las similitudes entre la pimienta y árboles forestales es que ambas proveen sombra al cacao, se aumenta la biodiversidad y el productor se beneficia por la venta de estos productos. Sin embargo; la pimienta se cosecha más fácil propiciando un mejor cuidado a las plantaciones de cacao, en contraste, los árboles forestales pueden provocar grandes pérdidas al momento de la cosecha y el cultivo del cacao podría resultar afectado al caer los arboles sobre las plantas de cacao.

\section{Proyecciones de cultivos: cacao, Pimienta y ÁRboles forestales}

En el cuadro 1 se muestran los precios del cacao, pimienta y árboles maderables para el estado de Tabasco que se han estudiado, y las proyecciones para el año 2015, 2018 y 2020. 
CUADRO 1.

Precios del cacao, pimienta y árboles maderables del periodo 2008-2014 con proyecciones para los años 2015, 2018 y 2020.

\begin{tabular}{llll}
\hline Año & $\begin{array}{l}\text { Cacao } \\
\text { seco } \\
(\$ / \mathrm{kg})\end{array}$ & $\begin{array}{l}\text { Pimienta } \\
\text { en } \\
\text { verde } \\
(\$ / \mathrm{kg})\end{array}$ & $\begin{array}{l}\text { Arbol } \\
\text { forestal } \\
(\text { m3r) }\end{array}$ \\
$\begin{array}{llll}\text { Diversas } \\
\text { especies }\end{array}$ \\
\hline 2008 & 25 & 14 & 2,804 \\
2009 & 31 & 10 & 3,767 \\
2010 & 37 & 15 & 2,685 \\
2011 & 40 & 17 & 3,130 \\
2012 & 38 & 16 & 3,705 \\
2013 & 36 & 14 & 2,512 \\
2014 & 38 & 10 & 2,782 \\
2015 & 40 & 11 & 2,696 \\
2018 & 46 & 18 & 2,323 \\
2020 & 49 & 20 & 1,887 \\
\hline
\end{tabular}

CONAFOR (2013), SIAP (2014)

\section{Pimienta}

En el sitio El Cometa, se registró la cantidad de $80 \mathrm{~kg}$ de cacao y $263 \mathrm{~kg}$ de pimienta, al precio de 36 y 11 pesos en verde por $\mathrm{kg}$ respectivamente. Lo cual genera el total de 5,773 pesos por la venta de cacao y pimienta, esto es lo que se espera para el año 2013. Manteniendo la producción constante del cacao y pimienta y multiplicándolo con los precios proyectados para los años 2015 y 2020 (cuadro 1), se obtendría un ingreso total de 6,093 y 9,180 pesos respectivamente.

\section{ÁRBOLES FORESTALES}

Los recursos generados al año por la venta de cacao fueron de $\$ 4,800$, sin embargo, este valor está en función de diversos factores, pero principalmente por el volumen de producción del mismo cultivo. En la actualidad es imposible tener un cálculo exacto pues depende del número de frutos cosechados por el productor, pues una parte se pierde por plagas, otras por enfermedades y otra por robo, pero en promedio se cosechan $120 \mathrm{~kg}$ por hectárea. Por otro lado, si existiese la necesidad de vender el arbolado presente en el cacaotal, se obtendría un ingreso de 332,256 pesos en madera de Caoba $(\$ 43,515)$, cedro $(\$ 25,172)$ y macuilí $(\$ 7,304)$. Dentro de las blandas Erytrina sp $(\$ 127,938)$, Ceiba $(\$ 77,469)$ y Jobo $(\$ 16,992)$. Otras 19 especies serian fuente importante de ingresos a escala menor (CONAFOR, 2014).

\section{ANÁLISIS Estadístico}

\section{DAP y altura}

Los resultados mostraron diferencias estadísticas significativas entre los árboles evaluados para el variable diámetro a la altura del pecho (DAP). El mayor valor promedio lo obtuvo la eritrina (Erythrina poeppigiana Walp. O. F. Cook) con $186.65 \mathrm{~cm}$., mientras el menor valor promedio lo registró la Casta Rica Blanca (Guarea sp.) con $15 \mathrm{~cm}$. En la evaluación de la altura, se encontraron diferencias estadísticas significativas 
entre las especies, obteniendo mayor valor promedio la eritrina con $30.76 \mathrm{~m}$ y el menor promedio la Casta Rica Blanca con $8.72 \mathrm{~m}$ (cuadro 2).

Con las evaluaciones realizadas, se observó que existe una correlación positiva entre el DAP y la altura de las especies, entre mayor sea el DAP mayor será la altura.

CUADRO 2.

Especies, diámetro a la altura del pecho (DAP), altura y tipo de madera de los árboles forestales registrados en el sitio La Ceiba del municipio de Comalcalco, Tabasco.

\begin{tabular}{|c|c|c|c|c|c|c|c|}
\hline $\begin{array}{l}\text { Tipo de } \\
\text { madera }\end{array}$ & Especies & Nombre Científico & Individuos & \begin{tabular}{|l|} 
DAP \\
promedio \\
(cm)
\end{tabular} & & $\begin{array}{l}\text { Altura } \\
(\mathrm{m})\end{array}$ & \\
\hline Blando & Eritrina & \begin{tabular}{|l|} 
Erythrina \\
poeppigiana.(Walp.) \\
O.F. Cook
\end{tabular} & 17 & 186.65 & a & 30.76 & $\mathrm{a}$ \\
\hline Blando & Ceiba & $\begin{array}{l}\text { Ceiba pentandra } \\
\text { (L.) Gaertn. }\end{array}$ & 8 & 157.50 & $a b$ & 30.50 & $a b$ \\
\hline Blando & Jobo & $\begin{array}{l}\text { Spondias mombin } \\
\text { L. }\end{array}$ & 15 & 106.80 & $\mathrm{bc}$ & 25.93 & $a b c$ \\
\hline Duro & Palo Sangre & Pterocarpus sp. & 6 & 95.83 & C & 25.33 & abcd \\
\hline Duro & Caimito & $\begin{array}{l}\text { Chrysophyllum } \\
\text { cainito L }\end{array}$ & 10 & 78.00 & $\mathrm{~cd}$ & 23.66 & abcde \\
\hline Semiblando & Guácimo & $\begin{array}{l}\text { Guazuma ulmifolia } \\
\text { Lam. }\end{array}$ & 20 & 76.20 & cde & 22.50 & bcde \\
\hline Blando & Palo Mulato & $\begin{array}{l}\text { Bursera simaruba } \\
\text { (L.) Rose. }\end{array}$ & 5 & 74.60 & cde & 22.10 & cde \\
\hline Duro & Mango & Mangifera indica L. & 7 & 73.00 & cdef & 20.80 & cdef \\
\hline Duro & Caoba & $\begin{array}{l}\text { Swietenia } \\
\text { macrophylla King. }\end{array}$ & 24 & 71.96 & cdef & 20.79 & cdef \\
\hline Duro & Candelero & $\begin{array}{l}\text { Cordia megalantha } \\
\text { S.F. Blake. }\end{array}$ & 6 & 71.20 & cdef & 18.40 & cdefg \\
\hline Duro & Teca & $\begin{array}{l}\text { Tectona grandis L. } \\
\text { F. }\end{array}$ & 8 & 67.13 & cdef & 18.00 & cdefg \\
\hline Blando & Mote & \begin{tabular}{|l|} 
Erythrina \\
americana Mill.
\end{tabular} & 12 & 61.17 & cdef & 17.62 & defgh \\
\hline Blando & Amatillo & $\begin{array}{l}\text { Ficus padifolia } \\
\text { H.B.K. }\end{array}$ & 6 & 58.33 & cdef & 16.93 & efghi \\
\hline Blando & Pochote & $\begin{array}{l}\text { Cochlospermum } \\
\text { vitifolium (Willd.) } \\
\text { Spreng. }\end{array}$ & 9 & 54.50 & cdef & 16.70 & efghi \\
\hline Duro & Naranja & Citrus sinensis L. & 7 & 53.29 & cdef & 16.66 & efghi \\
\hline Duro & Macuili & $\begin{array}{l}\text { Tabebuia rosea } \\
\text { (Bertol) DC. }\end{array}$ & 43 & 52.33 & cdef & 16.55 & efghi \\
\hline Semiblando & Cedro & $\begin{array}{l}\text { Cedrela odorata } \\
\text { (L.) Gaertn. }\end{array}$ & 44 & 49.93 & cdef & 13.66 & fghi \\
\hline Blando & Pochitoquillo & $\begin{array}{l}\text { Pleuranthodendron } \\
\text { mexicanum (A. } \\
\text { Gray) L.O. } \\
\text { Williams. }\end{array}$ & 7 & 36.29 & cdef & 13.50 & fghi \\
\hline Duro & Cocoite & $\begin{array}{l}\text { Gliricidia sepium } \\
\text { (Jacq.) Steud. }\end{array}$ & 36 & 34.39 & def & 12.71 & fghi \\
\hline Duro & $\begin{array}{l}\text { Palo } \\
\text { Colorado }\end{array}$ & $\begin{array}{l}\text { Simira } \\
\text { salvadorensis } \\
\text { Standl. }\end{array}$ & 17 & 23.00 & def & 11.85 & ghi \\
\hline Dudo & Ramoncillo & $\begin{array}{l}\text { Trophis racemosa } \\
\text { (L.) Urb }\end{array}$ & 6 & 17.50 & fe & 9.66 & hi \\
\hline Duro & $\begin{array}{l}\text { Casta Rica } \\
\text { Blanca }\end{array}$ & Guarea sp. & 11 & 15.00 & f & 8.72 & i \\
\hline
\end{tabular}

Nota Medias con letras diferentes en una misma columna difieren según prueba Tukey para $\mathrm{p}<0,05$. 
La eritrina es de los pocos árboles que puede tener el diámetro a la altura del pecho (DAP) cerca de los dos metros y con una altura mayor a los $25 \mathrm{~m}$, produce grandes cantidades de hojarasca y es fijadora de nitrógeno. La especie ayuda a conservar y mejorar el suelo y auxilia al aumento de rendimiento en cultivos asociados (generalmente perenes). La leña que produce esta especie es de baja calidad y no es recomendable para este uso.

Fuste limpio

Los resultados mostraron diferencias estadísticas significativas para los árboles evaluados en la variable fuste limpio. El mayor promedio fue para la eritrina (Erythrina poeppigiana Walp. O. F. Cook) con $20.11 \mathrm{~m}$ y el menor promedio para el árbol Ramoncillo (Trophis racemosa L. Urb) con $5.33 \mathrm{~m}$ (cuadro 3). 
CUADRO 3.

Evaluación de la variable fuste limpio de los diferentes árboles forestales en el sitio La Ceiba del municipio de Comalcalco, Tabasco.

\begin{tabular}{|c|c|c|c|c|}
\hline Especies & Nombre científico & $\begin{array}{l}\text { Total de } \\
\text { individuos }\end{array}$ & $\begin{array}{l}\text { Promedio } \\
\text { de fuste } \\
\text { limpio } \\
\text { (m) }\end{array}$ & \\
\hline Eritrina & $\begin{array}{l}\text { Erythrina } \\
\text { poeppigiana } \\
\text { (Walp.). O. F. Cook }\end{array}$ & 17 & 20.11 & $\bar{A}$ \\
\hline Caoba & $\begin{array}{l}\text { Swietenia } \\
\text { macrophylla King. }\end{array}$ & 24 & 17.20 & $\mathrm{Ab}$ \\
\hline Teca & $\begin{array}{l}\text { Tectona grandis L. } \\
\text { F. }\end{array}$ & 8 & 16.87 & $\mathrm{Ab}$ \\
\hline Ceiba & $\begin{array}{l}\text { Ceiba pentandra } \\
\text { (L.) Gaertn. }\end{array}$ & 8 & 15.75 & $a b c$ \\
\hline Palo Sangre & Pterocarpus sp. & 6 & 13.66 & abcd \\
\hline Macuili & $\begin{array}{l}\text { Tabebuia rosea } \\
\text { (Bertol) DC. }\end{array}$ & 43 & 13.32 & abcde \\
\hline Palo Mulato & $\begin{array}{l}\text { Bursera simaruba } \\
\text { (L.) Rose. }\end{array}$ & 5 & 13.20 & abcde \\
\hline Jobo & $\begin{array}{l}\text { Spondias mombin } \\
\mathrm{L} \text {. }\end{array}$ & 15 & 13.13 & abcde \\
\hline Cocoite & $\begin{array}{l}\text { Gliricidia sepium } \\
\text { (Jacq.) Steud. }\end{array}$ & 36 & 10.91 & bcdef \\
\hline Pochote & $\begin{array}{l}\text { Cochlospermum } \\
\text { vitifolium (Willd.) } \\
\text { Spreng. }\end{array}$ & 9 & 10.62 & bcdef \\
\hline Cedro & $\begin{array}{l}\text { Cedrela odorata } \\
\text { (L.) Gaertn. }\end{array}$ & 44 & 10.52 & bcdef \\
\hline Caimito & $\begin{array}{l}\text { Chrysophyllum } \\
\text { cainito L. }\end{array}$ & 10 & 9.70 & cdef \\
\hline Candelero & $\begin{array}{l}\text { Cordia megalantha } \\
\text { S. F. Blake }\end{array}$ & 6 & 9.40 & cdef \\
\hline Amatillo & $\begin{array}{l}\text { Ficus padifolia } \\
\text { H.B.K. }\end{array}$ & 6 & 9.00 & cdef \\
\hline $\begin{array}{l}\text { Palo } \\
\text { Colorado }\end{array}$ & $\begin{array}{l}\text { Simira } \\
\text { salvadorensis } \\
\text { Standl. }\end{array}$ & 17 & 9.00 & cdef \\
\hline Mote & $\begin{array}{l}\text { Erythrina } \\
\text { americana Mill. }\end{array}$ & 12 & 8.50 & def \\
\hline Mango & $\begin{array}{l}\text { Mangifera indica } \\
\text { L. }\end{array}$ & 7 & 7.80 & def \\
\hline Guácimo & $\begin{array}{l}\text { Guazuma } \\
\text { ulmifolia Lam. }\end{array}$ & 20 & 7.30 & def \\
\hline Naranja & Citrus sinensis L. & 7 & 7.14 & def \\
\hline Pochitoquillo & $\begin{array}{l}\text { Pleuranthodendron } \\
\text { mexicanum (A. } \\
\text { Gray) L.O. } \\
\text { Williams. }\end{array}$ & 7 & 6.42 & Ef \\
\hline $\begin{array}{l}\text { Casta Rica } \\
\text { Blanca }\end{array}$ & Guarea sp. & 11 & 5.45 & F \\
\hline Ramoncillo & $\begin{array}{l}\text { Trophis racemosa } \\
\text { (L.) Urb }\end{array}$ & 6 & 5.33 & $\mathrm{~F}$ \\
\hline
\end{tabular}

Nota Medias con letras diferentes en una misma columna difieren según prueba Tukey para $\mathrm{p}<0,05$.

Los resultados obtenidos se asemejan con el estudio realizado en Vargas, Venezuela; en donde se registraron 69 especies para reforestar. La especie eritrina fue la principal para este

proceso por tener un DAP y altura considerable (Camacho y Plonczak, 2012). Comparando estos valores con el presente estudio, puede inferirse que esta especie puede ser una opción para mejorar la productividad del sitio. 
Aunque en el sitio La Ceiba, no todas las especies registradas son de alto valor comercial, de acuerdo con Finegan (1992), en el futuro, estos sistemas agroforestales cobraran importancia para el suministro de recursos forestales debido a la baja oferta de maderas preciosas. Los SAF's crean mejores condiciones para el productor ya que obtiene recursos económicos, estimulan la acumulación de carbono y aumenta la diversidad de la vida silvestre. Destacan también especies con DAP y alturas similares a la eritrina, por ejemplo: Ceiba pentandra (L.) Gaertn, Spondias mombin L., Swietenia macrophylla King. y Cedrela odorata (L.) Gaertn.; estos tienen un alto valor comercial y son de utilidad para las comunidades rurales de Tabasco (Sánchez et. al. 2012) ya que el precio de la madera preciosa cada año aumenta y con ello mejoran las ganancias.

Otras especies como Trophis racemosa y Guarea sp., habitan debajo de ambientes sombreados pero los diámetros de sus fustes no desarrollan. Similar resultado se registró para Imataca, Venezuela, donde ambas especies se registraron ingresos pues maderas preciosas (Díaz et al. 2010), sin embargo, pueden ser utilizadas como cercas vivas, postes o ser utilizadas para leña.

\section{ConCLUSIón}

La sustitución de árboles de sombra muertos por árboles de pimienta representa una oportunidad de incremento en el ingreso económico a familias rurales dedicadas a la producción de cacao.

El Sistema Agroforestal cacao incrementará su valor económico de acuerdo al número de árboles de maderas preciosas presente en el mismo. La demanda de madera en el mercado nacional e internacional ha propiciado que los precios de la madera preciosa aumenten, por tal razón si el productor asocia el cultivo de cacao con especies maderables incrementara ingreso. La asociación de árboles de pimienta y árboles forestales en el cacaotal, constituye una oportunidad para diversificar los productos y beneficiar al productor, ya que en la actualidad los ingresos por la venta de pimienta son más al tos que los ingresos por la venta del cacao mismo.

\section{LITERATURA CITADA}

Banco Mundial. 2012. La promoción del empleo decente en el sector forestal para mejorar la nutrición y la seguridad alimentaria. Consultado: http://www.fao.org/docrep/018/mg489s/mg489s.pdf 14/03/2015

Bárcenas P. G. M. y Ordó-ez C. V. R. 2008. Calidad de la madera de los árboles de sombra. En: Manson V. R. H., Hernández O. V., Gallina S. y Mehltreter K. (eds) Agroecosistemas cafetaleros de Veracruz biodiversidad, manejo y conservación. INECOL, INE-SEMARNAT. 322 p.

Beer J. W., Muschler R., Kass D. and Somarriba E. 1998. Shade management in coffee and cacao plantations. Agroforestry Systems, 4: 175-189. https://doi.org/10.1007/978-94-015-9008-2_6

Beer J., Ibrahim M., Somarriba E., Barrance A. and Leakey R. 2004. Establecimiento y manejo de árboles en sistemas agroforestales. Capítulo 6. Árboles de Centroamérica. OFICATIE. $46 \mathrm{p}$

Camacho J. Y. and Plonczak M. 2012. Estudio fitosociológico de dos lotes en el piso de bosque premontado en la parroquia Carayaca, estado Vargas, Venezuela. Revista Forestal Venezolana, 56(1):62-72

Claridades Agropecuarias. 2001. Un Horizonte ASERCA del Mercado Agropecuario. Consultado: http:// www.infoaserca.gob.mx/claridades/revistas/098/ca098.pdf 22/02/2015

Comisión Nacional Forestal (CONAFOR). 2010. Reporte de precios de productos forestales. Trimestre IV de 2010. Consultado: http://www.conafor.gob.mx:8080/documentos/docs/39/4749Reporte\%20de\%20Precios\%20de \%20Productos\%20Forestales.pdf 05/05/2015

Comisión Nacional Forestal (CONAFOR). 2014. Precios de productos forestales maderables. Reporte trimestral Enero/Marzo 2014. Consultado: 05/05/2015. http://www.conafor.gob.mx:8080/ documentos/ docs/39/5239Reporte\%20de\%20Precios\%20de\%20Productos\%20Forestales.pdf 
Córdova A. V., Sánchez H. M., Estrella C. N. G., Macías L. A., Sandoval C. E., Martínez S. T. y Ortiz G. C. F. 2001. Factores que afectan la producción de cacao (Theobroma cacao L.) en el ejido Francisco I. Madero del Plan Chontalpa, Tabasco, México. Universidad y Ciencia, 17 (34): 93-100.

Dalquist R. M. Whelan M. P. Winowiecki L. Polidoro B. Candela S. Harvey C. A. Wulfhorst J. D. McDaniel P. A. and Bosque-Pérez N. A. 2007. Incorporating livelihoods in biodiversity conservation: a case study of cacao agroforestry systems in Talamanca, Costa Rica. Biodivers. Conserv. 16:2311-2333. https://doi.org/10.1007/ s10531-007-9192-4

De Almeida A. A., y Valle R. R. 2007. Ecophysiology of the cacao tree. Brazilian Journal of Plant Physiology, 19:425-448 https://doi.org/10.1590/S1677-04202007000400011

Deheuvels O., Avelino J., Somarriba E. and Malezieux E. 2012. Vegetation structure and productivity in cocoabased agroforestry systems in Talamanca, Costa Rica. Agric. Ecosyst. Environ. 149, 181-188. https:// doi.org/10.1016/j.agee.2011.03.003

Díaz P. W. A., Rueda J., Acosta O., Martinez O., Castellanos H. 2010. Composición florística del bosque ribere-o del río San José, Reserva Forestal de Imataca, Estado Bolívar, Venezuela. Revista: Acta Botánica Venezuelica. 33 (1):1-21

Dormon E. N. A., Van Huis A., Leeuwis C., Obeng-Ofori D., Sanky-Dawson O. 2004. Causes of low productivity of cocoa in Ghana: farmer's perspectives and insights from research and the socio-political establishment. NJAS Wageningen Journal of Life Sciences, 52 (3-4):237-259. https://doi.org/10.1016/S1573-5214(04)80016-2

FAOSTAT. 2014. Sistema Estadístico de la Organización de las Naciones Unidas para la Agricultura y la Alimentación. Consultado: http://faostat.fao.org/site/567/default.aspx. 22/03/15.

Finegan B. 1992. The management potential of neotropical secondary lowland rain forest. Forest Ecology and Management, 47 (1-4): 295-321 https://doi.org/10.1016/0378-1127(92)90281-D

Francis C. A. 1990. Potential of multiple cropping systems. p. 137-150. In: M. A. Altieri and S. B. Hecht (Eds.). Agroecology and Small Farm Development. CRC Pres Boca Raton. Boston PMid:2319584 PMCid:PMC1016938

García E. W. H. 1971. Posibilidad de desarrollo y cultivo de la pimienta gorda (Pimienta dioica) en el estado de Tabasco, Chapingo, México. 43 p.

González L. V. W. 2005. Cacao en México: competitividad y medio ambiente con alianzas. USAID. D.F. México. 93 p.

Greenberg R., Bichier P., Cruz-Anglon A. 2000. The conservation value for birds of cacao plantations with diverse planted shade in Tabasco, Mexico. Anim. Conserv. 3, 105-112. https://doi.org/10.1111/ j.1469-1795.2000.tb00235.x

Griffith D. M. 2000. Agroforestry: a refuge for tropical biodiversity after fire. Conserv. Biol. 14, 325-326. https:// doi.org/10.1046/j.1523-1739.2000.99101.x

Ibarra M. A. C., Arriaga W. S., y Estrada M. A. 2001. Avifauna asociada a dos cacaotales tradicionales en la región de la Chontalpa, Tabasco, México. Universidad y Ciencia, 34 (17)

Instituto Nacional de Estadística y Geografía (INEGI). 2012. Consultado: http://cuentame.inegi.org.mx/ monografias/informacion/tab/poblacion/ 12/05/2014

Instituto Nacional de Investigaciones Forestales, Agrícolas y Pecuarias (INIFAP). 2011. Programa estratégico para el desarrollo rural sustentable de la región sur - sureste de México: Trópico Húmedo 2011. Paquete Tecnológico Pimienta Gorda (Pimienta dioica L. Merril). Establecimiento y mantenimiento. Centro de Investigación Regional Golfo Centro. Campo experimental "El Palmar". Tezonapa, Veracruz. 56 pp.

Jefferson S. H., van Breugel M., Dylan J. C., Timothy G. G., Andrew P., Daisy H. D., Mark H. W., Emilio M. J. D., Diogenes I.N. C. and Mark S. A. 2011. Early growth and survival of 49 tropical tree species across sites differing in soil fertility and rainfall in Panama. Forest Ecology and Management 261(10):1580-1589 https:// doi.org/10.1016/j.foreco.2010.08.019

Marcos M. R. 2006. Análisis socioeconómico de viabilidad en el establecimiento y manejo de la Pimienta dioica. Universidad Veracruzana. Centro de Investigaciones Tropicales. Xalapa de Enríquez, Veracruz. 14 pp. 
Murgueitio E., Calle Z., Uribe F., Calle A., and Solorio B. 2011. Native trees and shrubs for the productive rehabilitation of cattle ranching lands. Forest Ecology and Management, 261:1654-1663. https:// doi.org/10.1016/j.foreco.2010.09.027

Organización de las Naciones Unidas para la Alimentación y la Agricultura (FAO). 2005. Situación de los bosques del mundo 2005. Consultado: http://www.fao.org/docrep/007/y5574s/y5574s00.htm. 12/02/14

Organización de las Naciones Unidas para la Alimentación y la Agricultura (FAO). 2014. Inventario forestal nacional. Manual de campo modelo. Programa de Evaluación de los Recursos Forestales (ERF). Guatemala. 89 p.

Ospina A. A. 2002. La Agroforestería: un saber popular. Unidad de Investigación Fundación Ecovivero 2 p. http:// www.ecovivero.org/Ecoarticuloabril.pdf. Consultado 12/02/14

Perez-Salicrup D. R. and Esquivel R. 2008. Tree infection by Hypsipyla grandella in Swietenia macrophylla and Cedrela odorata (Meliaceae) in Mexico's southern Yucatan Peninsula. Forest Ecology and Management, 255:324-327. https://doi.org/10.1016/j.foreco.2007.09.054

Ramírez M. A. 2009. Diversidad florística y macrofauna edáfica en plantaciones agroforestales de cacao en Cárdenas Tabasco. Tesis Maestría en Ciencias-Colegio de Postgraduado Campus, Tabasco. 86 p.

Ramos R. R. 2001. Análisis del uso sustentable de los suelos con plantaciones de cacao (Theobroma cacao L.) en Tabasco, México, aplicando sistemas de información geográfica. Tesis Maestría en Ciencias Colegio de Postgraduados Campus, Montecillo. Edo. México. 146 pp.

Roa R. H. A., Salgado M. M. G. y Álvarez H. J. 2009. Análisis de la estructura arbórea del sistema agroforestal de cacao (Theobroma cacao L.) en el Soconusco, Chiapas, México. Acta Biológica Colombiana (ABC). 14 (3): 97-110.

Sánchez D., Arends E., Villarreal A., y Serrano J. 2012. Composición florística de la regeneración natural en áreas de aprovechamiento forestal, Estación Experimental Caparo, Barinas-Venezuela. Revista Forestal Latino. 23:43

Sánchez L. J. A., y Dubón D. 1993. Algunos cultivos anuales asociados con cacao en un nuevo sistema de producción. Memoria. Editorial por W. Phillips M. Turrialba, C. R., CATIE. 69-79 pp.

Schroth G. and Harvey C. 2007. Biodiversity conservation in cocoa production landscapes. Biodivers Conserv 16: 2237-2244. https://doi.org/10.1007/s10531-007-9195-1

Servicio de Información Agroalimentaria y Pesquera (SIAP). 2014. Cierre de la producción agrícola por cultivo. Anuario Estadístico de la Producción Agrícola. Consultado: http://www.siap.gob.mx/index.php? option $=$ com_wrapper\&view $=$ wrapper\&Itemid $=184.03 / 11 / 2015$.

Sol S. A. 1999. Costos de producción de pimenta gorda o tabasco en el estado de Tabasco. Informe técnico 24 p.

Somarriba E. y Beer J. 1999. Sistemas agroforestales con cacao en Costa Rica y Panamá. Agroforestería en las Américas, $5(22): 7-11$.

Sotomayor A., García E., Gonzales M. y Lucero A. 2008. Modelos agroforestales. Sistema productivo integrado para una agricultura sustentable. Instituto Forestal de Chile (INFOR), Castilla 109 - C. 23 p.

Vázquez-Ya-es C., Bátiz M., Alcócer M. S., Gual M., y Sánchez D. 1999. Árboles y arbustos potencialmente valiosos para la restauración ecológica y reforestación. Reporte técnico del proyecto J084. CONABIO-Instituto de Ecología, UNAM. 129 pp.

Vega M. 2005. Planificación agroforestal participativa para el enriquecimiento de fincas cacaoteras orgánicas con especies le-osas perennes útiles en Alto Beni, Bolivia. Tesis Mag. Sc. Turrialba, CR. CATIE. 108 pp.

Zuidema P. A., Peter A. L, Liesje M., Wouter G. and Niel P. R. A. 2005. A physiological production model for cocoa (Theobroma cacao): Model presentation, validation and application. Agricultural Systems. 84:195-225 https:// doi.org/10.1016/j.agsy.2004.06.015 